\title{
CONTENUTO DI URANIO E TORIO NELLE METEORITI
}

\author{
M. Curatolo - D. Palumbo - M. Santangelo
}

Molti ricercatori in questi ultimi anni si sono interessati al problema della composizione delle meteoriti; dai risultati sperimentali si è cercato trarre elementi circa la genesi di questi materiali, questione ancora aperta, ed avere informazioni sui processi chimici e termodinamici cui le meteoriti furono soggette prima della loro caduta sulla terra.

Uno degli aspetti del problema è quello dell'abhondanza percentuale degli elementi chimici e della loro composizione isotopica nella materia meteorica dei diversi tipi: ciò perché esso è connesso con quello più generale dell'origine e distribuzione degli elementi nel cosmo e nella terra, nonché con quello dell'età delle meteoriti ( ${ }^{1}$ ).

In un recente lavoro Urey e collaboratori (-), esaminando un gran numero di analisi chimiche effettuate su questi materiali, sono pervenuti alla formulazione di alcuni criteri di classificazione in base alla percentuale dei componenti più abbondanti ed alla presenza o meno di disomogeneità strutturali nella massa fondamentale. Fra gli elementi meno abbondanti presentano particolare interesse quelli delle due famigrlie radioattive naturali Torio e Uranio: le loro concentrazioni sono state determinate per alcune meteoriti siliciche (stony meteorites) e per qualcuna ferrica (iron meteorites) $\left({ }^{3}\right)$.

Purtroppo però i valori trovati, con metodi diversi, scartano tra loro, anche per lo stesso tipo di campione, comunque se pure è azzardato fare estrapolazioni e dedurre conclusioni da dati ancora così in. certi, sono questi per il momento i pochi che si possono sfruttare per un tentativo di interpretare le possibili relazioni di costituzione e origine tra meteoriti e materiali terrestri.

I punti più delicati per la determinazione del contenuto di elementi radioattivi naturali nelle meteoriti, sono i seguenti:

1) basso contenuto di Uranio e Torio $\left(10^{-} \div 10^{-9}\right)$;

2) alta probabilità di contaminazione dei campioni;

3) inadeguatezza di alcuni metodi di misura.

Poiché nel nostro laboratorio, in questi ultimi tempi è stata im- 
piegata la tecnica delle emulsioni nucleari per determinazioni di basse attivita $\left({ }^{4}\right)$, abbiamo voluto eseguire con tale metodo, misure sulle meteoriti. Riferiamo qui di seguito sui risultati ottenuti.

La meteorite a nostra disposizione (") era un frammento di condrite che va sotto il nome di "Château Renard" descritta da A. Dut-

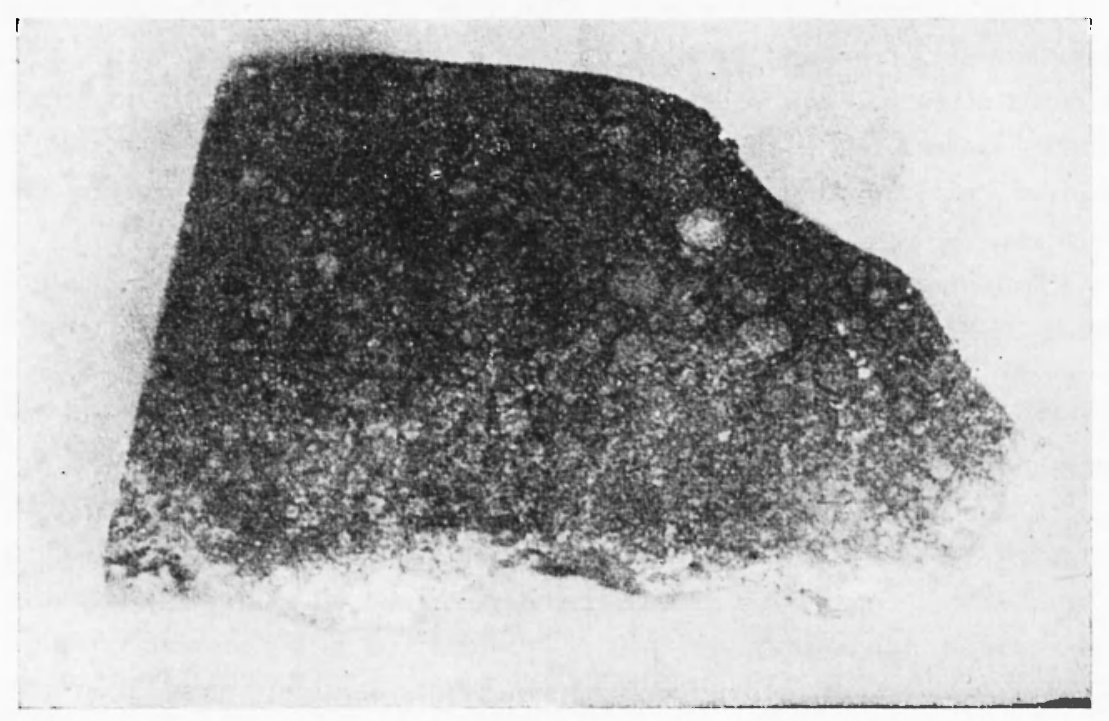

Fig. 1

freney $\left({ }^{5}\right)$, essa era in tutto simile per composizione chimica ed aspetto morfologico a quella investigata nel 1950 da Paneth e collaboratori ( ${ }^{6}$ ), denominata: "The Beddgelert meteorite ). In fig. 1 è mostrata la superficie del campione con ingrandimento $3 x$; in essa sono visibili i "condri ) caratteristici di questo tipo di meteorite. La densità è risultata $3,44 \mathrm{gr}_{\mathrm{g}} / \mathrm{cm}^{3}$. Il metodo di misura impiegato, come è stato accennato sopra, consisteva nello esporre una superficie, piana e perfettamente pulita, della meteorite ad una emulsione Ilford $C_{2} 50$ u ed al successivo esame degli spettri delle alfa.

Particolare cura si è dovuta avere per evitare reazioni chimiche sull'emulsione, provocate dal contatto dei materiali metallici (ferro)

1*) Ringraziamo il Prof. A. Bellanca, Direttore dell'Istituto di Mineralogia di Palermo che gentilmente ci ha fornito il campione da noi studiato. 
contenuti nel campione; nelle nostre esposizioni tra le due superficie - l lasciava interposto uno strato di aria di spessore noto. E poiché l'ativita da misurare era di valore molto basso (quasi al limite di sensibilita del metodol, sono state impiegate lastre freschissime e qualche volta sottoposte ad un processo chimico di sradicamento, in ogni caso per ogni esposizione veniva sempre determinato il fondo sia sulle latrine esposte al campione, sia su altre tenute insieme alle prime. Il tempo di esposizione è stato di circa 50 giorni. Nella tabella I, colonna 6, sono riportati i risultati sperimentali dello spettro delle proiezioni orizzontali delle $\alpha$, i numeri delle diverse righe danno le tracce le cui proiezioni orizzontali sono $\geqslant r$ (misurato in $\downarrow$. Poiché, come si è detto, nella esperienza si era lasciato uno strato daria tra superficie del campione ed emulsione, non è stato possibile applicare per l'interpretazione dello spettro sperimentale, le formule usuali, valevoli per il caso che emulsione e campione stiano perfettamente a contatto ${ }^{i}$ ).

Riguardo allo strato d'aria si possono fare due ipotesi:

a) che lo strato non contenga nuclidi attivi, cioè che sia trascurabile la diffusione di radon e toron dal campione verso l'esterno;

b) che vi sia una diffusione apprezzabile.

Nell'ipotesi limite a il problema dell'interpretazione dello spettro non presenta che qualche difficoltà di calcolo. Come è stato già mostrato in un precedente lavoro di uno di noi ( $\bar{i}$ ), fatta l'ipotesi semplificatrice che i rapporti tra i percorsi nell'aria, nell'emulsione e nel campione di una particella $\alpha$, siano indipendenti dall'energia, la distribuzione delle proiezioni orizzontali, per lelemento $i^{\text {mo }}$ della famiglia, è data dalla espressione:

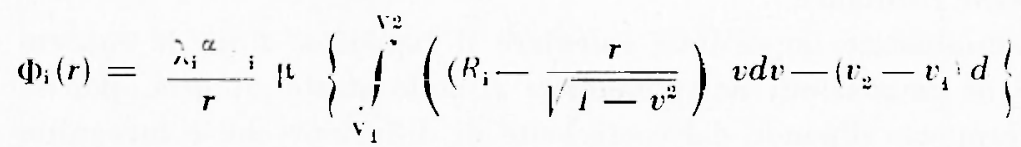

dove $\left(b_{i}(r) \grave{e}\right.$ il numero di tracce prodotte per $\mathrm{cm}^{-}$e per sec. dai nuclidi attivi alfa, della specie $i^{\text {ma }}$, che hanno proiezioni orizzontali $\geqslant r, \quad \lambda^{\alpha}$ la costante di disintegrazione $\alpha, n_{\mathrm{j}}$ il numero dei nuclidi per unita di volume, $\mu\left(^{*}\right.$ ) il rapporto (supposto costante) tra il percorso nell'emulsione e quello nella meteorite delle particelle $\alpha, R_{\mathrm{i}}$ il percorso totale nell'emulsione delle alfa di energia $E_{\mathrm{j}}, d$ lo spessore

(*) Il calcolo di $4 t$ è stato effettuato prendendo come base la composizione me. dia delle condriti. 
d'aria in unità equivalenti di spessore di materiale, $v_{1}$, e v.. le radici comprese tra 0 e 1 , della equazione:

$$
\left[R_{\mathrm{i}}-\frac{r}{\sqrt{1-v^{2}}}\right] v=d
$$

Risolvendo la [2], e sostituendo nella [1] (la quadratura è immediata), facendo la $\dot{\Sigma}\left(\phi_{\mathbf{i}}(r)\right.$ per tutti i nuclidi di ciascuna famiglia radioattiva, supposta in equilibrio, è stato calcolato lo spettro integrale delle proiezioni orizzontali delle tracce: nelle colonne 2 e 3 della tabella I figurano i numeri di tracce di proiezioni orizzontali $\geqslant r$ prodotte per $\mathrm{cm}^{2}$ e per sec. misurati in unità convenzionali calcolabili in funzione di $\mu$, rispettivamente delle famiglie dell'Uranio e Torio.

In questa ipotesi $a$ lo spettro sperimentale dovrebbe potersi ottenere come combinazione lineare delle colonne 2 e 3 della tabella $I$, con coefficienti proporzionali alle concentrazioni di $U$ e $T h$ (si è trascurato il contributo della famiglia dello Attinio). L'impossibilitì di ottenere un accordo, sia pure grossolano, tra i dati sperimentali e quelli calcolati, in particolare il numero relativamente alto delle tracce lunghe nella statistica sperimentale, mostra la inattendibilità dell'ipotesi.

Si è allora supposto (ipotesi $b$ ) che vi fosse una diffusione naturale di radon e toron, sicché le tracce osservate siano dovute in parte alle particelle alfa emesse dai nuclidi attivi contenuti nel materiale ed in parte a quelle emesse dalle emanazioni diffuse nello strato d'aria, nonché dai depositi attivi, che si sono supposti uniformemente distribuiti sulla superficie dell'emulsione e su quella della meteorite (praticamente, dato il piccolo valore di $d$, qualsiasi altra ipotesi porterebbe all'identico risultato).

E attualmente impossibile calcolare il rapporto $\sigma$ tra le concentrazioni di emanazioni nella metorite e nello strato di aria, poiché questo rapporto dipende dal coefficiente di diffusione che è incognito, e dalla forma geometrica, non certamente semplice, del volume di aria in cui avviene detta diffusione.

Comunque se si assume per $\sigma$ uno stesso valore per le due emanazioni, si può calcolare applicando la formula ( $\left.{ }^{\top}\right)$ :

$$
\Phi_{\mathrm{i}}(r) \smile \varphi_{\mathrm{i}}(r)-\frac{\lambda_{\mathrm{i}}^{\alpha} n_{\mathrm{i}}}{r} \sqrt{1-\left(\frac{r}{R_{\mathrm{i}}}\right)^{2}} d
$$

la distribuzione delle proiezioni orizzontali di tracce dovute ad $\alpha$ emesse dal Radon e Toron diffusi e dai loro depositi attivi. I risultati 
numerici sono riportati in tabella $I$, colonne 4 e 5 . Per comodità di calcolo detti valori sono stati calcolati assumendo per le emanazioni diffuse nello strato unità convenzionali 10 volte più piccole di quelle assunte per le concentrazioni analoghe nella meteorite.

Indicando allora con $C_{\mathrm{t}}$ e $C_{\mathrm{Th}}$ le concentrazioni (in unità convenzionali, di $U$ e $T h$ nella meteorite, con $\Phi_{u}(r), \Phi^{\prime}{ }_{u}(r), \Phi_{T_{\mathrm{h}}}(r)$, ()$^{\prime}{ }_{T h}(r)$ rispettivamente i dati delle colonne 2-5, lo spettro sperimentale $(\Phi)(r)$ dovrebbe essere rappresentato dalla equazione:

$$
\Phi(r)=C_{\mathrm{u}}\left[\Phi_{\mathrm{u}}(r) \cdot \rho \Phi_{\mathrm{u}}^{\prime}(r)\right]-C_{\mathrm{Th}}\left[\Phi_{\mathrm{Th}_{\mathrm{h}}}(r)-\rho \Phi_{\mathrm{Th}}^{\prime}(r)\right]
$$

in cui $c$ è proporzionale a $\sigma$.

Dalle [4] si dovrebbero poter ricavare $C_{\mathrm{u}},{ }{ }_{\mathrm{Th}} \in \in$. $\dot{\mathrm{E}}$ stato visto che la migliore approssimazione si ottiene supponendo $\bullet$ molto grande, cioe, praticamente trascurabile rispetto al contributo dei prodotti della diffusione, il contributo delle $\alpha$ emesse dei nuclidi attivi della meteorite. Data però l'accennata difficoltà di calcolare $\rho$, per mezzo delle [4] è stato possibile calcolare solo il rapporto $C_{\mathrm{Th}} / C_{\mathrm{u}}$, e non separatamente le due concentrazioni.

Detto rapporto risulta dai nostri dati sperimentali, calcolato dalle [3]:

$$
C_{\mathrm{Th}} / C_{\mathrm{u}}=\gamma=2,66
$$

É impossibile valutare lo scarto relativo a tale valore.

\section{TABElla I}

\begin{tabular}{|r|r|r|r|r|r|}
\hline$r$ in $\mu$ & $U$ & $T h$ & $R n$ & $T n$ & $\begin{array}{r}\text { Valori } \\
\text { sperim. }\end{array}$ \\
\hline & & & & & \\
\hline 5 & 1000 & 1000 & 631 & 494 & 2082 \\
10 & 502 & 641 & 482 & 402 & 816 \\
15 & 202 & 318 & 331 & 310 & 516 \\
20 & 82 & 144 & 167 & 202 & 291 \\
25 & 35 & 65 & 73 & 99 & 163 \\
30 & 10 & 32 & 40 & 52 & 72 \\
35 & - & 13 & 9 & 33 & 24 \\
40 & - & 2 & - & 17 & 7
\end{tabular}

Il valore di $\gamma$, da noi determinato per il campione di condrite, si accorda abbastanza bene con quelli misurati da altri ricercatori su meteoriti dello stesso tipo (vedi tab. II). C’è da notare peró che i 


\begin{tabular}{|c|c|c|c|c|c|c|c|c|c|c|c|c|}
\hline 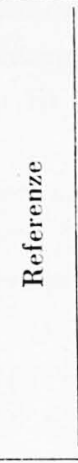 & & 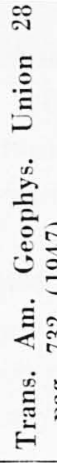 & & 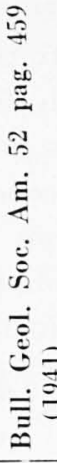 & 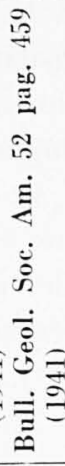 & & & 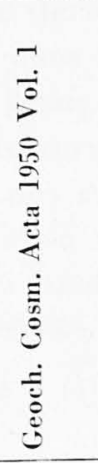 & 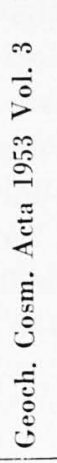 & 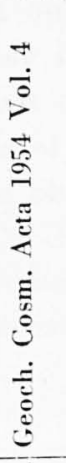 & 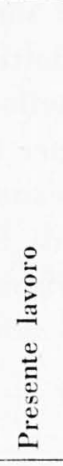 & 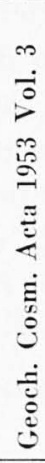 \\
\hline 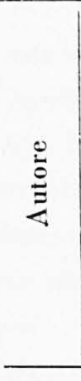 & & 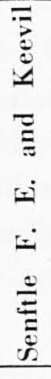 & $\begin{array}{l}\stackrel{0}{ } \\
\dot{z} \\
\dot{z}\end{array}$ & 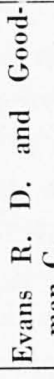 & 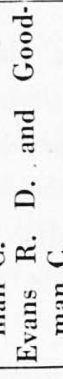 & & & 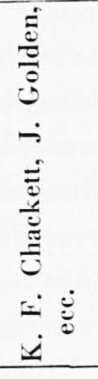 & 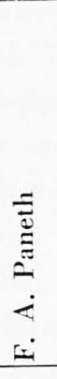 & 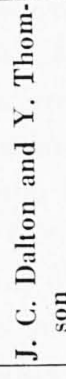 & $=\frac{\dot{d}}{\stackrel{0}{0}}$ & 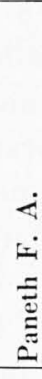 \\
\hline 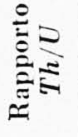 & & & $\begin{array}{l}\overrightarrow{b 0} \\
\dot{s}\end{array}$ & ণุ & $\stackrel{\varrho}{:}$ & $\stackrel{\vartheta}{\stackrel{0}{\sigma}}$ & & $\stackrel{2-2}{\circ}$ & 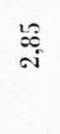 & is & $\begin{array}{c}b_{i} \\
\text { is }\end{array}$ & is \\
\hline 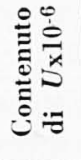 & & $\stackrel{0}{\circ}$ & $\begin{array}{c}\vec{b} \\
-i\end{array}$ & $\stackrel{-}{\dot{H}}$ & $\begin{array}{l}\stackrel{\circ}{\circ} \\
\Leftrightarrow\end{array}$ & $\stackrel{\mathscr{\infty}}{\Leftrightarrow}$ & & 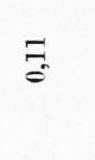 & 点 & $\begin{array}{l}\stackrel{0}{\circ} \\
\stackrel{-}{\circ}\end{array}$ & 1 & $\stackrel{5}{0}$ \\
\hline 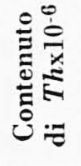 & & 崫 & $\stackrel{\tilde{a}}{\circ}$ & 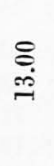 & $\vec{\phi}$ & 10 & & $\stackrel{\text { ڤે }}{\Leftrightarrow}$ & 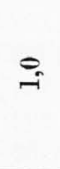 & $\begin{array}{l}\tilde{D} \\
\stackrel{0}{0}\end{array}$ & 1 & ت् \\
\hline & 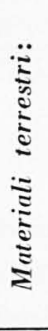 & 咅 & 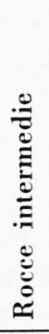 & 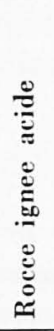 & 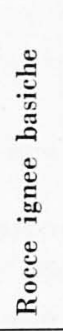 & 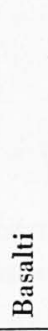 & 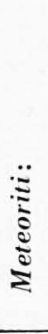 & 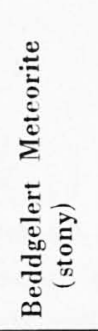 & 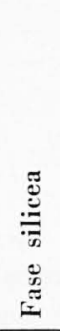 & 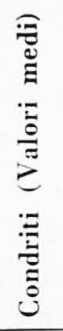 & & 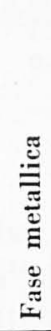 \\
\hline
\end{tabular}


valori assoluti del contenuto di $U$ e $T h$ scartano di molto a seconda delle diverse tecniche impiegate ( $\left.{ }^{*}\right)$, solo il rapporto $C_{\mathrm{T}}, C_{\mathrm{u}}$ si conserva entro limiti piuttosto ristretti, esso si approssima al valore trovato per le rocce ignee acide della crosta terrestre Igraniti, rocce intermedie).

Per la fase metallica delle meteoriti si hamo pochi dati e quindi non ha molto senso stabilire raffronti; si puó solamente dire che oltre al fatto, già diverse altre volte riscontrato, del loro più basso contenuto di $U$ e $T h$, il rapporto $\gamma$ per questi materiali cresce, cosi come si osserva per le rocce basaltiche. Tutto questo potrebbe forse avere qualche attinenza con le ipotesi che si fanno sullorigine delle meteoriti: H. Brown e Cl. Patterson, seguendo altra via, da un raffronto quantitativo per alcuni elementi contenuti nei materiali terrestri e nelle meteoriti, arrivano ad affermare che queste ultime provengono da qualche pianeta con caratteristiche fisiche e chimiche simili a quelle della terra. Il nostro risultato non è in contrasto con questa ipotesi.

Palermo - Istituto di Fisica dellUniversità - Maggio 1954.

\section{RIASSUNTO}

Si riferisce su una misura del rapporto tra lo concentrazioni di $\mathrm{Th}$ e $\mathrm{\textrm {T }}, \mathrm{C}_{\mathrm{n}}, \mathrm{C}_{\mathrm{a}}$, in una meteorite silicica (condrite) eseguita con il metodo delle emulsioni nucleari. Mettendo a raffronto il risultato con $i$ valori trovati da altri ricercatori, impiegando tecniche diverse, si ha un discreto accordo. Si fa notare come il valore $\mathrm{C}_{\mathrm{Th}} / \mathrm{C}_{\mathrm{u}}$ delle meteoriti siliciche (stony meteorites) si approssima a quello dei materiali della crosta terrestre.

\section{$S U M M A R Y$}

The $\mathrm{C}_{\mathrm{Th}} \mathrm{C}_{\mathrm{u}}$ ratio betuen the Th-and $\mathrm{U}$-concentration of a stonymeteorite has been measured with the nuclear-emulsion method. There is not detectable discrepancy between this value and the others obtained with different techniques. It is emphasized that the $\mathrm{C}_{\mathrm{Th}} / \mathrm{C}_{\mathrm{u}}$ ratio in the meteorites approximates the same ratio in the materials of the crust. 


\section{BIBLIOGRAFIA}

(1) Browx H. and Pattenson C., Journal Geology, Vol. 55, pag. 405 e pag. 508 (19.17). Journal Geology, vol. 56. pag. 85 (19.18).

Bonto G. Geochimica el Cosmochimical Acti, Vol. 6, n. 5-6, pag. 209 (1951).

Thomson S. J. Marne K. I., Geoch. Cosm. Acta, Vol. 7, pag. 169 (1955).

Arrol W. J. Jicobi R. B. and Paneth F. A., Nature, 119, 235 (19.12).

Pitrersox (., Geoch. Cosm. Acta, vol, 7, pag. 151 (1955).

(2) Uney C. H. and Cratg H. Geochimica Cosm. Acti, vol. 1, pag. 36 (1953).

(ङ) Quirke T. T. and Fiskeisteix L., Am. Journ. Sci., Vol. H. pag. 237 (1917).

DAvis G. L. Am. Journ. Sci, Vol. 245, pag. 6?7 (19.47); Am. Journ. Sci., Vol. 218, pag. 107 (1950).

Dalton J. C., Golmen J., Martin G. R., Merchi E. R. and Thouson S. J., Geochimica Cosm. Acta, Vol. 3, pag. 272 (1953).

Dalton J. C. and Thonson S. J. Geochim. Cosm. Acta, Vol. 5, pag. 71 (195.1).

Paneth F. A., Geoch. Cosm. Acti, Vol. 3, 257 (1953).

(4) Barbera L, Curatolo M, Iniovisa M. M., Saxtangelo M., Amn. Geof., Vol. V, pag. 603 (1952) e Vol. VI, paig. 161 (1953).

(5) Dufreney A, Comptes Rendus 13, ti (19-11).

(6) Chacketr K. F. Golden J Mercer E. R, Paneth F. A. and Reasbeck P., Geoch. Cosm. Acta l, 3 (1950).

(T) Palunbo D., Ann. Geof., Vol. VI, n. 2, pag. 229 (1953).

(8) Pattersos C., Brows H., Tilton G., Inghman M., Phys. Rev., 92, 5 (1953). 